\title{
Estimation of an Improved Spectrum Sensing Threshold for Cognitive Radio using Smoothed Pseudo Wigner-Ville Distribution
}

\author{
W. O. Ajadi \\ Electrical and Computer \\ Engineering Department, \\ Ahmadu Bello University, \\ Zaria, Nigeria
}

\author{
S. M. Sani \\ Electrical and Computer \\ Engineering Department, \\ Ahmadu Bello University Zaria, \\ Nigeria.
}

\author{
A. M. S. Tekanyi \\ Electrical and Computer \\ Engineering Department, \\ Ahmadu Bello University \\ Zaria, Nigeria.
}

\begin{abstract}
Cognitive radio (CR) has been suggested as the solution to spectrum scarcity due to the fixed allocation employed worldwide by regulatory bodies. In order to avoid interference to a primary user signal, the CR has to be aware about the spectrum usage in the geographic area in which it wants to operate. The process of spectrum sensing is a fundamental task for obtaining this awareness and the result of this process determines the successful operation of cognitive radio. Energy detection is one of the methods of spectrum sensing with the lowest computational complexity but with low performance at low signal to noise ratio. Exploring energy detection has led to the application of many techniques one of which is the use of time-frequency analysis. This method employs distribution techniques for analyzing the energy spectral density of an observed signal with a view to setting a sensing threshold. However, the distribution techniques that were used in literature suffered from the problem of cross-terms which affect the analysis of the resulting distribution thereby leading to poor sensing performance at low signal-to-noise ratio. Smoothed pseudo Wigner-Ville distribution of the time-frequency analysis has been employed in this work to reduce the effect of crossterms for better sensing threshold. Simulation results evaluate the performance of the employed technique compared to pseudo Wigner-Ville for AWGN, Rician and Rayleigh channel conditions.
\end{abstract}

\section{Keywords}

Cognitive radio, Spectrum sensing, Time-Frequency Analysis, Smoothed Pseudo Wigner-Ville distribution, Cross-terms.

\section{INTRODUCTION}

This static spectrum allocation strategy has led to many successful applications like broadcasting and mobile communication and it has also led to almost the entire prime available spectrum being assigned for various applications [1]. It may thus seem that there is little or no spectrum available for emerging wireless products and services.

There had been several studies and reports over the years that showed that the static allocated spectrum was in fact vastly underutilized. A report presenting statistics regarding spectrum utilization showed that even during the high demand period of a political convention such as the one held in 2004 in New York City, only about 13\% of the spectrum opportunities were utilized [2]. Further, measurement on radio frequency bands from $30 \mathrm{MHz}$ to $910 \mathrm{MHz}$ was done in Mexico City of San Luis Potosi and showed 11.83\% [3],
Kwara State of Nigeria at $48.5 \mathrm{MHz}$ to $880 \mathrm{MHz}$ showed $12.02 \%$ usage in the urban areas [4], and also at $2.4 \mathrm{GHz}$ to $2.7 \mathrm{GHz}$ showed $22.56 \%$ usage in the urban areas [5], thus, all showing that spectrum was in fact underutilized. These findings also suggest that devices using advanced radio and signal processing technology should be able to exploit underutilized spectrum. Much of the early motivation for cognitive radio technology was indeed to accomplish such opportunistic spectrum use and to also alleviate the artificial scarcity of prime spectrum. This technology will revolutionize the way spectrum is allocated worldwide, the challenging task is carrying out reliable spectrum sensing at low signal-to-noise ratio (SNR) as the successful operation of CR depends on the result of spectrum sensing. This means that a secondary user needs to reliably detect a primary user that is transmitting at very low power or that is located far from the detection point. The selection of appropriate spectrum sensing technique should take into account the trade-off between the performance and the computational burden [6].

\section{SIMILAR WORKS}

There has been several works on spectrum sensing and the most common methods employed are energy detection, cyclostationary feature detection and matched filtering [7]. Energy detection has received most attention from researchers owing to its less computational complexity and non-requirement of the primary user's information before detecting its presence. The work of [8] used time-frequency analysis and employed Wigner-Ville distribution to detect the energy of the primary user but suffers the problem of cross-terms. Compressed sensing was used with pseudo Wigner-Ville distribution in [9] but the issue of cross-terms persisted. Wavelet transform was treated as a filter bank in [10] where multiple filters divided in high pass and low pass were used, this has and increased computational complexity. Also the work of [11] used pseudo Wigner-Ville distribution as a radio mode identification of the primary user signal, this technique comes with increased computational complexity. In [12] a hardware testbed that employ Wigner-Ville transform was implemented and in [13], a joint timefrequency transform with S-method was employed for spectrum sensing sake and achieved a good sensing threshold but with additional computation. This work aimed at improving the achievable sensing threshold while maintaining low computational complexity by employing smoothed pseudo Wigner-Ville distribution (SPWVD) to suppress the effects of cross-terms in the use of timefrequency techniques. 


\section{USE OF TIME-FREQUENCY ANALYSIS FOR SPECTRUM SENSING}

Using time-frequency analysis allows for analyzing the energy components of non-stationary signals in time and frequency domains simultaneously. Localizing signals in time and frequency domains simultaneously requires that the distribution techniques be a bilinear function. This bilinear function follows a quadratic superposition principles [14] defined as:

$W_{x+y}(t, v)=W_{x}(t, v)+W_{y}(t, v)+2 \mathcal{R}\left\{W_{x, y}(t, v)\right\}$

But,

$W_{x, y}(t, v)=\int_{-\infty}^{+\infty} x\left(t+\frac{\tau}{2}\right) y *\left(t-\frac{\tau}{2}\right) e^{-j 2 \pi v t} d \tau$

$2 \mathcal{R}\left\{W_{x, y}(t, v)\right\}$ is the cross-term present in the resulting multicomponent signal $W_{x+y}(t, v)$.

where: signal ' $x$ '

$W(t, v)$ denote the Wigner-Ville distribution of

$$
\begin{aligned}
& \text { ' } t \text { ' is the time } \\
& \text { ' } \tau \text { ' is a delay in time or time shift } \\
& \text { ' } v \text { ' is a delay in frequency or Doppler shift }
\end{aligned}
$$

In order to suppress the effects of cross-terms on the signal of analysis for better judgement of the energy spectral component, this work employed SPWVD which gives us the ability to smoothen out the cross-terms present without much compromise on the resolution. The SPWVD is given by:

$S P W_{x}(t, v)=\int_{-\infty}^{\infty} h(\tau) \int_{-\infty}^{\infty} g(v-t) s\left(t+\frac{\tau}{2}\right) s^{*}(t-$
$\left.\frac{\tau}{2}\right) d s e^{-j 2 \pi v t} d \tau$

$h(t)$ and $g(s-t)$ are the separate smoothing functions along the time and frequency domains respectively.

\section{METHODOLOGY}

A non-cooperative single secondary user was considered for this work. The scenario depicted a single secondary user scanning for spectrum holes opportunity at a target primary user band.

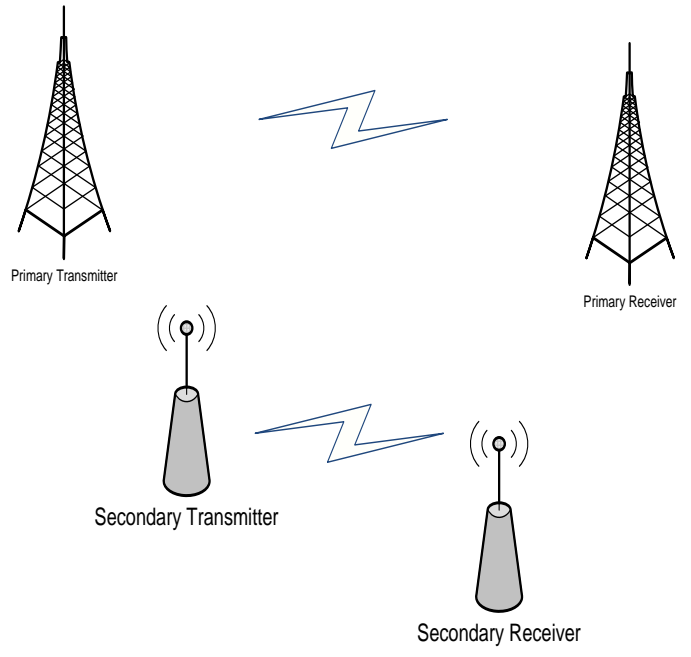

Figure 1 Spectrum sensing scenario for a primary user and secondary user
A bitmap grayscale image was used in this work, it was converted to a baseband signal, modulated and transmitted through the communication channel modeled under three different channel conditions of AWGN, Rayleigh, and Rician. The expression for one OFDM symbol starting at $t=t_{s}$ is given as [15]:

$$
\begin{aligned}
& S(t)=\operatorname{Re}\left\{\sum_{i=-\frac{N_{S}}{2}}^{\frac{N_{S}}{2}-1} d_{i+\frac{N_{S}}{2}} \exp \left(j 2 \pi\left(f_{c}-\frac{i+0.5}{T}\right)\left(t-t_{s}\right)\right)\right\} \\
& t_{s} \leq t \leq t_{s}+T
\end{aligned}
$$

where $d_{i}$ are complex modulation symbols, $i$ is the OFDM symbol number, $N_{S}$ is the number of subcarriers, $T$ the symbol duration, and $f_{c}$ the carrier frequency. The OFDM signal was simulated using Matlab.

The table 1 below summarizes the parameters used for the simulation of the OFDM signal transmission.

Table 1 OFDM Simulation Parameters

\begin{tabular}{|l|l|}
\hline Parameters & Values \\
\hline Source data & $\begin{array}{l}\text { Gray scale image with different sizes } \\
\text { (800 by 600,600 by 800, 400 by 300) }\end{array}$ \\
\hline IFFT size & 2048 \\
\hline $\begin{array}{l}\text { Number of } \\
\text { carriers }\end{array}$ & $\begin{array}{l}\text { Defined by(number of carrier } \leq \\
\text { ifft size } \\
2\end{array}-2$ ). \\
\hline $\begin{array}{l}\text { Modulation } \\
\text { method }\end{array}$ & $\begin{array}{l}\text { Varied between BPSK, QPSK, 16PSK, } \\
\text { 256PSK }\end{array}$ \\
\hline $\begin{array}{l}\text { Amplitude power } \\
\text { clipping (dB) }\end{array}$ & $3-9$ \\
\hline $\begin{array}{l}\text { Signal to Noise } \\
\text { Ratio (dB) }\end{array}$ & Varied between -45 to 0 \\
\hline
\end{tabular}

The simulation was done in two stages: (i) a scenario where there is an image been transmitted through the noisy channel and (ii) a scenario where nothing is been transmitted. The signal of transmission was then captured and analyzed by first performing Hilbert transform on it and then using SPWVD on it where the cross-terms are been suppressed without compromising poor resolution. This distribution is as given:

$$
\begin{aligned}
& S P W_{x}(t, v)=\int_{-\infty}^{\infty} h(\tau) \int_{-\infty}^{\infty} g(v-t) s\left(t+\frac{\tau}{2}\right) s^{*}(t- \\
& \left.\frac{\tau}{2}\right) d s e^{-j 2 \pi v t} d \tau
\end{aligned}
$$

The Kaiser-Bessel window was used for the smoothing window $h(t)$ and its beta parameter used was 7 to reduce the effects of side lobes and the window length $M$ of 79 was used to achieve a clear distinction between closely packed frequencies of the signal in the frequency plane. The Blackman window was used for the smoothing window $g(s-t)$ with window length $\mathrm{M}$ of 33 gave a satisfactory cross-terms suppression in the time plane.

The energy spectral density of the smoothed distribution was extracted using the marginal time-frequency function of the time-frequency tool box. The energy spectral densities were extracted as [16]:

$E=\int_{-\infty}^{+\infty} \int_{-\infty}^{+\infty} t f r(t, f) d f d t$

where $E$ represents the energy spectral density of the distribution $t f r$ represents the Smoothed Pseudo WignerVille distribution function $t$ represents the vector containing 
time sample in seconds $f$ represents the vector containing frequency samples in hertz.

Target SNR of $-25 \mathrm{~dB}$ was used to determine the threshold because the standardization body specified that, the detection threshold for energy detection that can detect reliably at a received power of $-116 \mathrm{dBm}$ at the receiver is required below which reliable detection is not guaranteed. The energy spectral densities at $-25 \mathrm{~dB}$ after one hundred simulations were averaged in order to estimate and determine the threshold.

\section{PERFORMANCE METRICS}

The following are some of the performance metrics of interest:

1. Probability of False Alarm (PFA): The probability of false alarm is a measure of how many times the CR has identified the presence of a primary user in a channel whereas it does not exist. This is important from a secondary user perspective as it leads to losing transmission opportunity.

2. Probability of Missed Detection (PMD): The probability of missed detection is a measure of how many times the secondary user failed to detect the primary user. This is important from a secondary user perspective as it indicates how many times the CR fails to identify the presence of a primary user, while actually it does exist which will cause interference on the primary user's transmission.

3. Signal to Noise Ratio: SNR is important from a comparison point of view and explains the behavior of the spectrum sensing tool under varying noise conditions at specific receiver sensitivity. Most often this is measured at the output of the receiver.

\section{RESULTS AND DISCUSSION}

The plots in figures 2 to 7 show the performance of the thresholds obtained from both the smoothed pseudo WignerVille distribution (SPWVD) against the pseudo Wigner-Ville distribution (PWVD). The probabilities of missing detection were plotted against Signal to Noise Ratios (SNRs) and the probabilities of false alarm against SNRs for each channel condition modeled that is, AWGN, Rician and Rayleigh channels respectively.

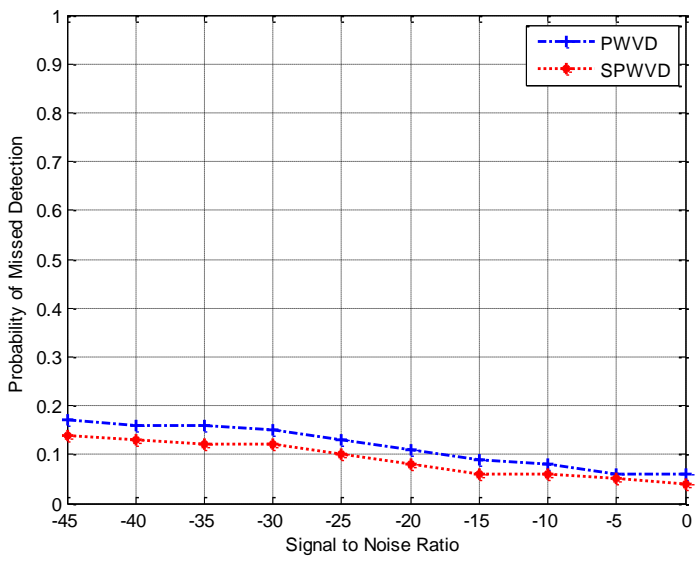

Figure 2 Plot of PMD against SNR for AWGN Channel

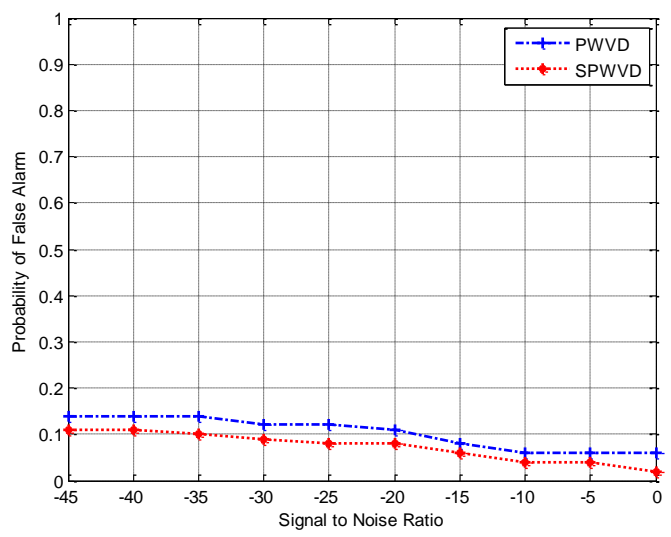

Figure 3 Plot of PFA against SNR for AWGN Channel

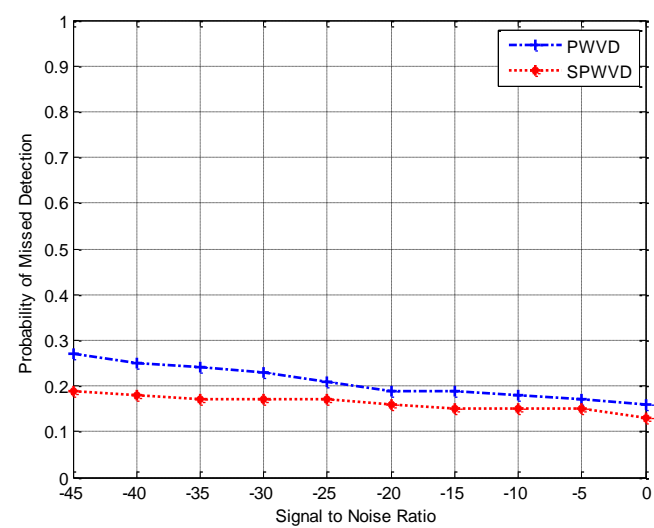

Figure 4 Plot of PMD against SNR for Ricain channel condition

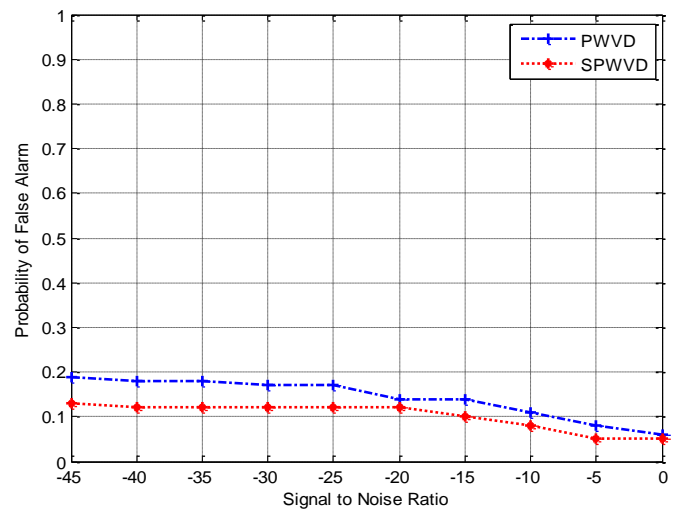

Figure 5 Plot of PFA against SNR for Rician channel condition

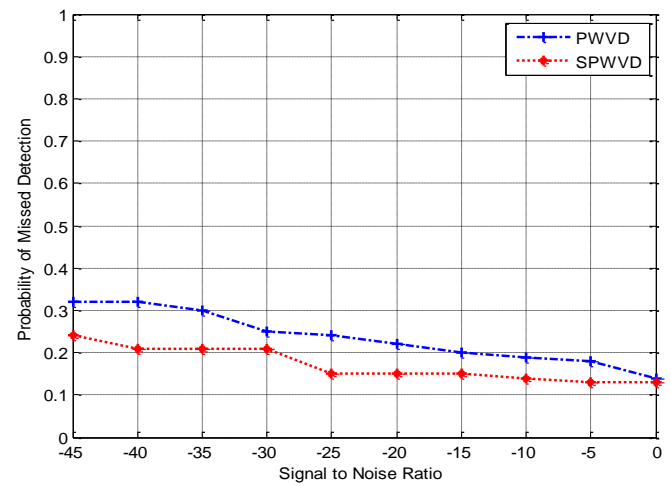

Figure 6 Plot of PMD against SNR for Rayleigh channel condition 


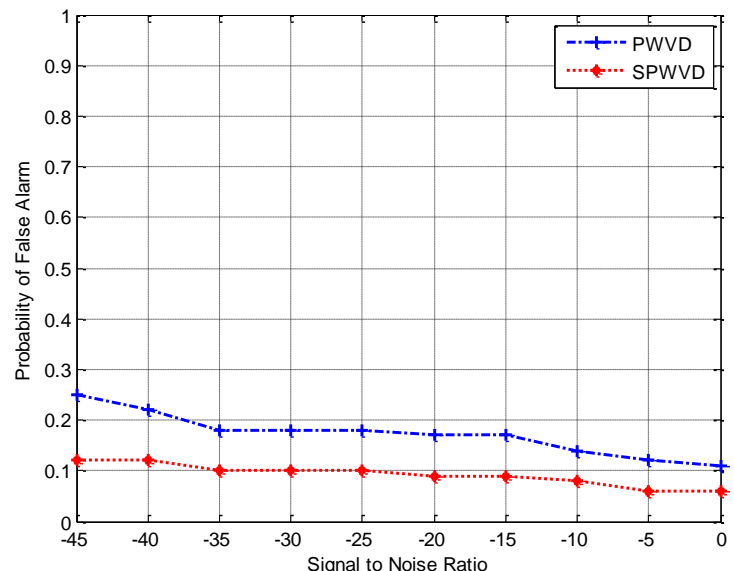

Figure 7 Plot of PFA against SNR for Rayleigh Channel Condition

\section{CONCLUSION}

The smoothed pseudo Wigner-Ville distribution (SPWVD) technique has been utilized in this work to reduce the effect of cross-term components by way of employing two smoothening filters for filtering the cross-terms generated while seeking to analyze OFDMA user signal in both time and frequency domain simultaneously. SPWVD yields a better sensing threshold when compared with Pseudo Wigner-Ville Distribution (PWVD) distribution. OFDMA user signal transmissions were simulated and analyzed in MATLAB R2013b environment and the time frequency toolbox that contains the time-frequency analysis techniques developed at CNRS were used to achieve the aim of this work. The determined threshold in the SPWVD technique was compared with that of PWVD for performance analysis and the SPWVD showed a superior performance in the respective channel conditions (AWGN, Rician and Rayleigh).

\section{REFERENCES}

[1] Biglieri, E., Goldsmith, A. J., Greenstein, L. J., Mandayam, N. B., \& Poor, H. V. (2012). Principles of cognitive radio: Cambridge University Press.

[2] McHenry, M., \& McCloskey, D. (2004). New York City spectrum occupancy measurements september 2004. Shared Spectrum Company, www. sharedspectrum. com.

[3] Aguilar-Gonzalez, R., Cardenas-Juarez, M., Rico, U. P., \& Stevens-Navarro, E. (2013). Power Spectrum Measurements from $30 \mathrm{MHz}$ to $910 \mathrm{MHz}$ in the City of San Luis Potosi, Mexico. Procedia Technology, 7, 3036.

[4] Babalola, O., Garba, E., Oladimeji, I., Bamiduro, A., Faruk, N., Sowande, O., Muhammad, M. (2015). Spectrum occupancy measurements in the TV and CDMA bands. Paper presented at the 2015 International Conference on Cyberspace (CYBER-Abuja).
[5] Ayeni, A. A., Faruk, N., Bello, O. W., Sowande, O. A., Onidare, S. O., \& Muhammad, M. Y. (2016). Spectrum Occupancy Measurements and Analysis in the 2.4-2.7 $\mathrm{GHz}$ Band in Urban and Rural Environments. International Journal of Future Computer and Communication, 5(3), 142.

[6] Angrisani, L., Betta, G., Capriglione, D., Cerro, G., Ferrigno, L., \& Miele, G. (2014). Proposal and analysis of new algorithms for wideband spectrum sensing in cognitive radio. Paper presented at the 2014 IEEE International Instrumentation and Measurement Technology Conference (I2MTC) Proceedings.

[7] Vaidehi, G., Swetha, N., \& Sastry, P. N. (2015). Entropy based Spectrum Sensing in Cognitive Radio Networks. Entropy, 4(11).

[8] Javed, F., \& Mahmood, A. (2010). The use of time frequency analysis for spectrum sensing in cognitive radios. Paper presented at the 2010 4th International Conference on Signal Processing and Communication Systems (ICSPCS)

[9] Monfared, S. S., Taherpour, A., \& Khattab, T. (2013). Time-frequency compressed spectrum sensing in cognitive radios. Paper presented at the Global Communications Conference (GLOBECOM), 2013 IEEE.

[10] Bektas, C., Akan, A., \& Odabasioglu, N. (2012). Energy based spectrum sensing using wavelet transform for fading channels. Paper presented at the 2012 4th International Congress on Ultra-Modern Telecommunications and Control Systems and Workshops (ICUMT)

[11] Javed, F., Shafi, I., \& Mahmood, A. (2012). A novel radio mode identification approach for spectrum sensing in cognitive radios. International Journal of Communication Networks and Information Security, 4(2), 86 .

[12] Biagi, M., Rinauro, S., Colonnese, S., Scarano, G., \& Cusani, R. (2014). WiVCoRA: Wigner-Ville Cognitive Radio Access for Secondary Nodes. Vehicular Technology, IEEE Transactions on, 63(9), 4248-4264.

[13] Hiremath, S., Patra, S., \& Mishra, A. (2015). Spectrum Sensing for Cognitive Radio using S-method based Joint Time-Frequency Representation.

[14] Boashash, B. (2015). Time-frequency signal analysis and processing: a comprehensive reference: Academic Press.

[15] Ingram, D. M. A., Acosta, G., \& Matlab, O. S. U. (2000). Smart Antenna Research Laboratory. Guillermo Acosta August.

[16] Auger, F., Flandrin, P., Goncalves, P., \& Lemoine, O. (2005). Time-Frequency Toolbox for use with MATLAB. Retrieved from http://tftb.nongnu.org/ 